\title{
INFLUENCE OF CARDANOL ENCAPSULATED ON THE PROPERTIES OF POLY(LACTIC ACID) MICROPARTICLES
}

\author{
Jaciene J. F. Cardoso ${ }^{\mathrm{a}, \mathrm{b}}$, Eduardo Ricci-Júnior ${ }^{\mathrm{c}}$, Denise Gentilid, Luciana S. Spinelli ${ }^{\mathrm{a}, \mathrm{e}}$ and Elizabete F. Lucas ${ }^{\mathrm{a}, \mathrm{e}, *}$ \\ anstituto de Macromoléculas, Universidade Federal do Rio de Janeiro, Av. Horácio Macedo 2030, Cidade Universitária, 21941 - \\ 598 Rio de Janeiro - RJ, Brasil \\ 'Departamento de Tecnologia Química, Universidade Federal do Maranhão, Av. dos Portugueses S/N, Campus do Bacanga, 65080- \\ 540 São Luís - MA, Brasil \\ 'Faculdade de Farmácia, Universidade Federal do Rio de Janeiro, Av. Carlos Chagas Filho, 373, Bl. L, Cidade Universitária, Ilha \\ do Fundão, 21941-590 Rio de Janeiro - RJ, Brasil \\ ${ }^{d}$ Centro para Educação Tecnológica Celso Suckow da Fonseca, Av. Maracanã, 229, Maracanã, 20271-110 Rio de Janeiro - RJ, Brasil \\ eInstituto Alberto Luiz Coimbra de Pós-Graduação e Pesquisa em Engenharia, Universidade Federal do Rio de Janeiro, Av. Horácio \\ Macedo, 2030, Bls. F e G, Ilha do Fundão, 21941-598, Rio de Janeiro - RJ, Brasil
}

Recebido em 08/08/2017; aceito em 07/11/2017; publicado na web em 04/12/2017

\begin{abstract}
In this study, microparticles of poly(lactic acid) (PLA) were produced to encapsulate cardanol, varying molar mass of the polymer matrix, concentration of the emulsifier (PVA) and concentration of the chemical additive (cardanol). The droplet size distribution, polydispersity index, morphology, the interaction between cardanol and PLA, cardanol encapsulation efficiency and release profile of this chemical additive were assessed. The morphological characterization showed that the microparticles containing cardanol were presented as microcapsules up to a maximum cardanol concentration limit that could be incorporated. The addition of cardanol during the production of the microparticles led to an increase in the average diameter of the microparticles obtained, both those with low and high molar mass (MPLA3 and MPLA100, respectively), with the increase depending on the quantity of cardanol incorporated. DSC results showed a shift in melting point and a change in Tg and Tm with the incorporation of cardanol, suggesting an interaction between cardanol and PLA matrix. Higher encapsulation efficiency and slower release of cardanol were achieved when using microparticles with higher molar mass (MPLA100). The microparticles of MPLA100/1PVA/50C provided the slowest additive release among the formulations tested. Therefore, the processes for encapsulation and controlled release of cardanol in the PLA matrix were efficient.
\end{abstract}

Keywords: microparticles; controlled release; cardanol; poly(lactic acid).

\section{INTRODUCTION}

Cardanol is an inexpensive, renewable, natural organic material obtained by vacuum distillation of cashew nut shell liquid, with a wide range of applications. ${ }^{1,2}$ It is a phenolic compound with an aliphatic chain of 15 carbons that are meta-substituted in an aromatic ring, giving it important chemical and physico-chemical characteristics, mainly due to its amphiphilic character. Cardanol is a complex mixture of 3-n-pentadecyl phenol, 3-(n-pentadeca-8-enyl) phenol, 3-(n-pentadeca-8,11-dienyl) phenol, and 3-(n-pentadeca-8,11,14trienyl) phenol. ${ }^{3,4}$

Due to its chemical similarity with nonylphenol (a synthetic chemical additive with proven action as an asphaltene stabilizer), ${ }^{5}$ cardanol has been tested for its ability to stabilize asphaltenes in crude oil, both in its original form and after undergoing step-growth or chain-growth polymerization, demonstrating good performance compared to nonylphenol. ${ }^{4,6-8}$ Therefore, the substitution of nonylphenol by cardanol can have both environmental and economic advantages because cardanol is obtained from a renewable source and is cheaper to produce.

The petroleum industry uses a wide range of chemical additives to overcome the challenges of extraction, production, processing, transport and storage of crude oil. ${ }^{9-12}$ In some situations, the application of the chemical additive requires shutting down production, causing significant economic losses. ${ }^{13,14}$ Therefore, the development of systems

*e-mail: elucas@metalmat.ufrj.br for controlled release of chemical additives is very attractive to optimize certain operations. The incorporation of active agents through polymer matrixes with controlled release of additives is widely used in several industrial sectors. The advantages over conventional systems for inclusion of chemical additives are: a) more effective inclusion, with progressive and controlled release of the additive as the matrix degrades, b) significant reduction of toxicity due to control of the optimal period for the additive to remain in the medium, c) greater safety (less risk of negative reactions with other substances present in the fluid) and convenience (lower number of additive injections), d) more accurate guidance to specific targets without affecting other active species; and e) greater flexibility in the inclusion of additives, because both hydrophilic and lipophilic substances can be incorporated. ${ }^{15,16}$ The medical/pharmaceutical, food, cosmetic, veterinary and agroindustrial segments are among the areas where controlled release of additives is used. The greatest number of studies of controlled release of additives have been published in the medical/pharmaceutical field. ${ }^{17-20}$ The studies examining applications in the petroleum industry have been target of researchers. ${ }^{21,22}$ There is a number of patents that describe systems for controlled release of additives in the oil industry such as, emulsionbased systems for release of antifoam agents (with various applications besides the oil industry) and release of additives in cement (for oil well servicing), as well as cross-linked polymers for the controlled release of biocides in oil fields. ${ }^{23-25}$

Many polymers, both natural (chitosan, hyaluronic acid, alginic acid) and synthetic can be used as the polymer matrix for encapsulation of active agents. The most widely studied synthetic polymers for this 
purpose are poly(hydroxy acids), polyanhydrides, polyols and aliphatic polyesters, among others. These polymers are used more often because their degradation rates can be varied from hours to months depending on the synthesis conditions, characteristics of each polymer, composition (in the case of copolymers) and conditions of the environment where they will be applied. ${ }^{16,26}$ Among the various types of degradable aliphatic polyesters, poly(lactic acid) (PLA), poly (glycolic acid) (PGA) and their copolymer poly(D, L-lactic acid-co-glycolic acid) (PLGA) have been tested and approved for use in controlled release systems. ${ }^{27}$ PLA has been used as a matrix in controlled release systems for many applications (medical/pharmaceutical, food, cosmetic, veterinary and agroindustrial segments) because of its attractive combination of characteristics, such as biodegradability, biocompatibility and thermoplastic behavior, among others. ${ }^{28-30}$ Studies of controlled release systems generally involve the preparation of microparticles containing a polymer matrix and an encapsulated additive. This additive can be released gradually, according to the needs of each application. ${ }^{21}$ Microparticles can be classified according to their structure into microcapsules or microspheres. Microspheres are microparticles consisting of a solid polymer matrix, where the additive is uniformly dispersed or solubilized. Microcapsules, unlike microspheres, form a system that acts as a reservoir, where a layer of polymer covers the additive (encapsulated material or core) with variable thickness. ${ }^{31}$ Recent studies ${ }^{21,32}$ have shown that the degradation profile of PLA powder and microparticles varies significantly in function of the medium, having been tested in aqueous, saline and organic media, all of which are commonly used in the petroleum industry. It has been showed that in an organic medium (e.g., toluene), PLA is dissolving instead of degrading allowing the release of the encapsulated additive. In any event, PLA has the advantage of being degradable in relatively short time periods under specific conditions, making it an environmentally friendly material. ${ }^{21}$

However, the formation of the microparticles, their encapsulation efficiency and their release behavior from the polymer matrix are also influenced by the formulation and concentration of the encapsulated additive, aspects that have not been widely studied for the specific range of conditions in the oil industry.

In this study, PLA microparticles were produced in the presence of cardanol, varying the molar mass of the base polymer, the concentration of the emulsifier (PVA) and the concentration of the chemical additive (cardanol). The characteristics of the microparticles formed, the interaction between base polymer and chemical additive and the release profile of the additive were investigated.

\section{EXPERIMENTAL}

\section{Materials}

Poly(lactic acid) (PLA), Ingeo 2002, supplied by Nature Works (Minnetonk, USA), with specification $\mathrm{M}_{\mathrm{w}}=107,000 \mathrm{~g} \mathrm{~mol}^{-1}{ }^{21}$ and poly(lactic acid), synthesized by direct polycondensation, with $\mathrm{M}_{\mathrm{w}}=3,500 \mathrm{~g} \mathrm{~mol}^{-1}{ }^{21}$ are identified as PLA100 and PLA3, respectively. Poly(vinyl alcohol) (PVA), with $\mathrm{M}_{\mathrm{w}}=13,000-23,000$ $\mathrm{g} \mathrm{mol}^{-1}$ and hydrolysis degree $=87-89 \%$, was supplied by Sigma Aldrich Chemistry, São Paulo, Brazil. Cardanol was acquired from Satya Cashew Chemicals, Tamil Nadu, India. Chloroform P.A. and dichloromethane P.A. were supplied by Tedia Brasil, Rio de Janeiro, Brazil. All the materials were used as received.

\section{Production of the PLA microparticles}

The PLA microparticles were prepared using the technique of emulsification followed by solvent evaporation. The aqueous phase was composed of an aqueous solution of PVA at concentration of 0.75 or 1.0 $\mathrm{wt} / \mathrm{v} \%$. The organic phase was prepared using $200 \mathrm{mg}$ of PLA, $6 \mathrm{~mL}$ of dichloromethane (organic solvent) and 0,25,50 or $100 \mathrm{mg}$ of cardanol, until dissolution of the constituents. To promote contact between the phases, the organic phase was added dropwise in the aqueous phase under stirring for 5 minutes at 6,000 rpm with an IKA Ultra-Turrax T18 Basic device, until the formation of an emulsion. Then the solvent was evaporated in a Büchi R-114 rotary evaporator. After evaporation, the microparticles were washed with distilled water, centrifuged at $5110 \mathrm{xg}$ (Beckman Coulter Avanti J-25 centrifuge with JA-25.50 rotor), frozen in a standard freezer and dried in a Liotop K105 lyophilizer.

The microparticles were produced using PLA3 or PLA100. Table 1 summarizes the composition of the microparticles and the respective nomenclature.

\section{Characterization of the polymeric microparticles containing cardanol}

The microparticles were characterized by thermogravimetric analysis (TGA), differential scanning calorimetry (DSC) and scanning electron microscopy (SEM), and their size and size distribution and encapsulation efficiency (EE) were evaluated.

The variation of mass loss of the material in function of temperature (thermogravimetric analysis) was assessed under a continuous $\mathrm{N}_{2}$ flow in the temperature range of 20 to $700{ }^{\circ} \mathrm{C}$ and heating rate of $20{ }^{\circ} \mathrm{C}$ $\mathrm{min}^{-1}$, in a TA Instruments Q500 thermogravimetric analyzer.

The microparticles were analyzed by differential scanning calorimetry (DSC) in a nitrogen atmosphere at a flow of $50 \mathrm{~mL}$ $\mathrm{min}^{-1}$ and temperature range of 10 to $250{ }^{\circ} \mathrm{C}$, with heating rate of $10{ }^{\circ} \mathrm{C} \mathrm{min}{ }^{-1}$. These analyses were carried out with a TA Instruments DSC-Q1000 calorimeter, under $\mathrm{N}_{2}$ atmosphere, previously calibrated with an indium standard.

The morphology of the dried microparticles was studied by scanning electron microscopy (SEM) with a JEOL JSM-561L microscope, operating at $15 \mathrm{KV}$. A small quantity of the sample (powder or microparticle) was fixed to a metal support and covered with a fine layer of gold under vacuum for about 1 minute.

The size and size distribution of the polymeric microparticles were determined in a Malvern MasterSizer 2000 laser diffraction particle size analyzer, which can measure the distribution of particles with diameters in the range from 0.02 to 2,000 $\mu \mathrm{m}$. For this purpose, a small quantity of particles was dispersed in distilled water using ultrasound and stirring at rotation of $1,600 \mathrm{rpm}$, in the device itself, to assure absence of agglomerates and passage of the laser beam through the suspension of dispersed particles. The parameters obtained by this technique were average particle diameter - $\mathrm{D}(\mathrm{v}$, $0.5)$, which represents the cutoff size at which $50 \%$ of the sample's particles are smaller, average volumetric diameter - D $(4,3)$, which represents the diameter of a sphere with the same average volume of the microparticles that compose the sample, and the polydispersity index (or span), which provides information on the homogeneity of the size distribution. ${ }^{33}$

\section{Encapsulation efficiency (EE)}

The quantity of cardanol present in the microparticles was determined by ultraviolet-visible (UV-Vis) spectrophotometry. A calibration curve (absorbance vs. concentration) of cardanol in dichloromethane was constructed with a Jasco V-630 spectrophotometer, using a quartz cell with $10 \mathrm{~mm}$ optical path at a wavelength of $270 \mathrm{~nm}$ (the wavelength of maximum absorption of cardanol in dichloromethane against a blank solution of PLA in dichloromethane). 
Table 1. Compositions and names of the microparticles

\begin{tabular}{|c|c|c|c|}
\hline Polymer & $\begin{array}{c}\text { PVA concentration } \\
(\mathrm{wt} / \mathrm{V} \%)\end{array}$ & Cardanol (mg) & Microparticles \\
\hline \multirow{8}{*}{ PLA3 } & \multirow{4}{*}{0.75} & 0 & MPLA3/0.75PVA/0C \\
\hline & & 25 & MPLA3/0.75PVA/25C \\
\hline & & 50 & MPLA3/0.75PVA/50C \\
\hline & & 100 & MPLA3/0.75PVA/100C \\
\hline & \multirow{4}{*}{1.0} & 0 & MPLA3/1PVA/0C \\
\hline & & 25 & MPLA3/1PVA/25C \\
\hline & & 50 & MPLA3/1PVA/50C \\
\hline & & 100 & MPLA3/1PVA/100C \\
\hline \multirow{8}{*}{ PLA100 } & \multirow{4}{*}{0.75} & 0 & MPLA100/0.75PVA/0C \\
\hline & & 25 & MPLA100/0.75PVA/25C \\
\hline & & 50 & MPLA100/0.75PVA/50C \\
\hline & & 100 & MPLA100/0.75PVA/100C \\
\hline & \multirow{4}{*}{1.0} & 0 & MPLA100/1PVA/0C \\
\hline & & 25 & MPLA100/1PVA/25C \\
\hline & & 50 & MPLA100/1PVA/50C \\
\hline & & 100 & MPLA100/1PVA/100C \\
\hline
\end{tabular}

$\mathrm{M}=$ microparticle; PLA $=$ poly $($ lactic acid $) ;$ PVA = poly $($ vinyl alcohol $)$ $\mathrm{C}=$ cardanol

About $10 \mathrm{mg}$ of microparticles containing the additive were dissolved in $20 \mathrm{~mL}$ of dichloromethane. Dichloromethane was the solvent due its capacity to break the microcapsules solubilizing the PLA and the additive incorporate. This dispersion was maintained by ultrasound for 10 minutes until complete breakdown of the microparticles in the solvent. Then the solution was filtered and the absorption was measured in the UV-Vis spectrophotometer at $270 \mathrm{~nm}$. Using the calibration curve, it was possible to obtain the additive concentration in the $10 \mathrm{mg}$ of dissolved microparticles. The encapsulation efficiency (\%) was estimated by correlating the quantity of the additive initially included in the formulations and that found in the solution after extraction for each mg of microparticles. The additive concentration was estimated as the percentage weight (wt/wt $\%$ ) of the active agent found in the microparticles. The encapsulation efficiency (EE\%) was determined by Equation 1.

$$
\mathrm{EE}(\%)=\frac{\text { Quantity of encapsulated additive }}{\text { Quantity of added additive }} \times 100
$$

\section{Evaluation of the cardanol release profile of the microparticles}

The microparticles were evaluated regarding their cardanol release profile in an organic solvent chloroform $\left(\mathrm{CHCl}_{3}\right)$. This solvent does not absorb at the same maximum wavelength as cardanol and PLA, unlike toluene which is the best solvent for this system since it is used as a petroleum model. Therefore, chloroform $\left(\mathrm{CHCl}_{3}\right)$, with a solubility parameter near that of toluene, was used in this test.

The method employed for this purpose was similar to that carried out to calculate the encapsulation efficiency, utilizing a response curve of cardanol in chloroform obtained by UV-Vis spectrophotometry at a wavelength of $270 \mathrm{~nm}$. For the release test, $10 \mathrm{mg}$ of microparticles was weighed in flasks and $20 \mathrm{~mL}$ of chloroform was added. The flasks were sealed and placed under constant agitation on a bar and magnetic shaker (MAG 15, Marte Equipamentos). The absorbance values were read at predetermined intervals $(15,30,45,60,90,120$ and $150 \mathrm{~min})$ at $270 \mathrm{~nm}$ in a Jasco V-630 spectrophotometer. The concentration and percentage of cardanol released to the medium in function of time (min) were determined using the previously obtained response curve.

\section{RESULTS AND DISCUSSION}

The results reported here of encapsulation of cardanol in systems formed of microparticles previously prepared and characterized in the presence of cardanol are groundbreaking. ${ }^{21}$ However, all the results regarding the microparticles without cardanol have been previously reported $^{21}$ and are presented here for the purpose of comparing the influence of the cardanol concentration on the microparticles' characteristics.

\section{Evaluation of the thermal behavior of the microparticles by thermogravimetric analysis (TGA) and differential scanning calorimetry (DSC)}

Thermogravimetric analysis (TGA) and differential scanning calorimetry (DSC) were used to ascertain, respectively, the thermal stability of the samples and the thermal transition behavior of the systems as well as the possible interactions of the additive with the polymer matrix. Table 2 reports the initial mass loss temperature $\left(\mathrm{T}_{\text {onset }}\right)$, obtained from the TGA curves, and the temperature of maximum mass loss or maximum degradation $\left(\mathrm{T}_{\max }\right)$, obtained from the DTG curves of the microparticle systems (PLA3 ${ }^{21}$, PLA100 ${ }^{21}$ ) and the pure samples ( $\mathrm{PVA}^{21}$ and cardanol).

As found in the previous study mentioned above, ${ }^{21}$ the pure polymer with low molar mass (PLA3), as expected, presented lower $\mathrm{T}_{\text {onset }}\left(255^{\circ} \mathrm{C}\right.$ ) than that of the polymer with high molar mass (PLA100, $\left.345^{\circ} \mathrm{C}\right)$. The thermal stability of the microparticles accompanied that of the respective polymers used to prepare them: the microparticles produced with the polymer having high molar mass (MPLA100) had higher thermal stability than those produced with the polymer having low molar mass (MPLA3).

Table 2. Onset temperature by TGA and temperature at maximum degradation by DTG of PLA3, ${ }^{21}$ PLA100, ${ }^{21} \mathrm{PVA},{ }^{21}$ cardanol and the obtained microparticles

\begin{tabular}{|c|c|c|c|c|}
\hline \multirow[t]{2}{*}{ Samples } & \multirow{2}{*}{$\begin{array}{l}\text { Onset } \\
\left({ }^{\circ} \mathrm{C}\right)\end{array}$} & \multicolumn{3}{|c|}{$\begin{array}{c}\text { Temperature at the maximum } \\
\text { degradation }\left({ }^{\circ} \mathrm{C}\right)\end{array}$} \\
\hline & & $1^{\text {st }}$ step & $2^{\text {nd }}$ step & $3^{\text {rd }}$ step \\
\hline PLA3 $^{21}$ & 255 & 332 & - & - \\
\hline $\mathrm{PVA}^{21}$ & 290 & 111 & 309 & 430 \\
\hline Cardanol & 233 & 273 & & \\
\hline MPLA3/0.75PVA/0C ${ }^{21}$ & 265 & 302 & - & - \\
\hline MPLA3/0.75PVA/25C & 255 & 285 & - & - \\
\hline MPLA3/0.75PVA/50C & 197 & 213 & 291 & - \\
\hline MPLA3/1PVA/0C 21 & 254 & 285 & - & - \\
\hline MPLA3/1PVA/25C & 253 & 281 & 356 & - \\
\hline PLA $100^{21}$ & 345 & 366 & - & - \\
\hline MPLA100/0.75PVA/0C ${ }^{21}$ & 336 & 366 & - & - \\
\hline MPLA100/0.75PVA/25C & 294 & 233 & 320 & 366 \\
\hline MPLA100/0.75PVA/50C & 288 & 230 & 313 & 362 \\
\hline MPLA100/0.75PVA/100C & 285 & 236 & 297 & - \\
\hline MPLA100/1PVA/0C ${ }^{21}$ & 279 & 318 & 361 & - \\
\hline MPLA100/1PVA/25C & 267 & 311 & - & - \\
\hline MPLA100/1PVA/50C & 251 & 294 & - & - \\
\hline MPLA100/1PVA/100C & 276 & 246 & 286 & - \\
\hline
\end{tabular}


In comparing the samples produced with polymer PLA3 against each other, the formation of microparticles promoted a reduction of the thermal stability, which was best observed at the temperature of maximum degradation of the first stage. Besides this, the addition of cardanol caused a change in the degradation profile of the samples. Pure PLA and PLA + PVA presented only one degradation stage, while the systems containing cardanol underwent up to three degradation stages. This behavior is related to the greater thermal susceptibility of cardanol in relation to the polymers (PLA and PVA). The effect was more pronounced at higher cardanol concentrations. Furthermore, for low cardanol concentrations, degradation was also observed at temperatures related to decomposition of the pure polymer. However, for higher concentrations, the degradation of the cardanol promoted a reduction in the degradation temperature of the polymer, so that the microparticles loaded with the additive degraded completely at lower temperatures than the microparticles made of pure polymer. Probably due the stronger interaction between cardanol and the polymers stabilizing the microparticles. In any event, all the degradation onset temperatures were well above the maximum temperatures found under normal conditions for application of additives in the petroleum industry.

The presence of PVA had little influence on the degradation onset temperature in relation to the pure polymer. The reason for this behavior is that PVA has three degradation stages (Table 2). The greatest variation was observed for the systems containing PLA3 and $1 \mathrm{wt} / \mathrm{v} \%$ of PVA, which started degrading at a temperature 47 ${ }^{\circ} \mathrm{C}$ lower than the pure PLA3. Nevertheless, these variations were smaller than those caused by adding cardanol.

In the field of micro-encapsulation, DSC is used to assess the crystallinity degree of the materials used, as well as to check for the existence of interactions between the additive and polymer matrix. ${ }^{34}$

As presented in a previous work, ${ }^{21}$ no endothermic or exothermic event was observed in the temperature range studied (-50 to 100 ${ }^{\circ} \mathrm{C}$ ) for MPLA3 microparticles. The DSC curves referring to the first heating of the samples of pure PLA100 and microparticles of the MPLA100/0.75PVA and MPLA100/1PVA groups are shown in Figures $1 \mathrm{a}$ and $1 \mathrm{~b}$, respectively.

It has been reported the analyses of the DSC curves for the empty microparticles (without cardanol). The incorporation of cardanol in the microparticles shifted the melting peaks to lower temperatures than observed initially for PLA100 and the empty microparticles. ${ }^{21}$ The higher the cardanol concentration in the microparticles was, the greater this shift was. This behavior suggests that an interaction occurred between the polymer and cardanol, since when a material undergoes a change in its physico-chemical state or reacts chemically, heat associated with this alteration is absorbed or released. This behavior can be better observed from the data in Table 3, which reports the $\mathrm{T}_{\mathrm{g}}$ (glass transition temperature), $\mathrm{T}_{\mathrm{m}}{ }^{(\text {a) }}$ (melting temperature at the $1^{\text {st }}$ first heating), $\Delta \mathrm{H}_{\mathrm{m}}{ }^{\text {(a) }}$ (enthalpy variation at the $1^{\text {st }}$ heating) and $\mathrm{T}_{\mathrm{m}}$ (melting temperature) of the different systems.

Table 3. Result of $\mathrm{T}_{\mathrm{g}}$ (glass transition temperature), $\mathrm{T}_{\mathrm{m}}{ }^{(a)}$ (melting temperature at the $1^{\text {st }}$ first heating), $\Delta \mathrm{H}_{\mathrm{m}}{ }^{\text {(a) }}$ (melting enthalpy variation at the $1^{\text {st }}$ heating) and $\mathrm{T}_{\mathrm{m}}$ (melting temperature) obtained by DSC for microparticles produced from PLA100 with and without cardanol

\begin{tabular}{lcccc}
\hline Samples & $\mathrm{T}_{\mathrm{g}}\left({ }^{\circ} \mathrm{C}\right)$ & $\mathrm{T}_{\mathrm{m}}\left({ }^{\circ} \mathrm{C}\right)^{\mathrm{a}}$ & $\Delta \mathrm{H}_{\mathrm{m}}\left(\mathrm{J} \mathrm{g}^{-1}\right)^{\mathrm{a}}$ & $\mathrm{T}_{\mathrm{m}}\left({ }^{\circ} \mathrm{C}\right)$ \\
\hline PLA100 $^{21}$ & 54 & $151 / 156$ & 38 & 153 \\
MPLA100/0.75PVA/0C & 44 & $148 / 155$ & 29 & 136 \\
MPLA100/0.75PVA/25C & 22 & 148 & 28 & 125 \\
MPLA100/0.75PVA/50C & 11 & 148 & 31 & 118 \\
MPLA100/0.75PVA/100C & 18 & 145 & 21 & 119 \\
MPLA100/1PVA/0C 21 & 44 & $148 / 155$ & 32 & 140 \\
MPLA100/1PVA/25C & 19 & 148 & 30 & 124 \\
MPLA100/1PVA/50C & 11 & 148 & 17 & 113 \\
MPLA100/1PVA/100C & 10 & 146 & 22 & 119 \\
\hline
\end{tabular}

(a) events of first heating.

The DSC results revealed that the glass transition temperature $\left(\mathrm{T}_{\mathrm{g}}\right)$ values of the PLA100 and the microparticles with and without cardanol were different, probably due to the different compositions of the samples and the intermolecular interaction between the components. The $\mathrm{T}_{\mathrm{g}}$ of the PLA100 declined from $54{ }^{\circ} \mathrm{C}$ to 44 ${ }^{\circ} \mathrm{C}$ after formation of the empty microparticles containing 0.75 and $1 \mathrm{wt} / \mathrm{v} \%$ of PVA. This reduction was in general higher as the cardanol concentration in the microparticles increased, and was most accentuated in the microparticles produced with $1 \mathrm{wt} / \mathrm{v} \%$ of PVA.

The appearance of a melting temperature $\left(\mathrm{T}_{\mathrm{m}}\right)$ indicates the presence of crystallinity in the material. The $T_{m}$ value is directly related to the interactions of cardanol with the polymer matrix, and its measurement can allow identifying the effects caused by the quantity of cardanol contained in the structure of the PLA microparticles. These effects can influence the microparticles' properties, such as encapsulation efficiency and release profile. As can be seen in Table 3, for the systems containing both $0.75 \mathrm{wt} / \mathrm{v} \%$ and $1 \mathrm{wt} / \mathrm{v} \%$ of $\mathrm{PVA}$, the $\mathrm{T}_{\mathrm{m}}$ value declined with increasing cardanol concentration. Moreover, as observed for $\mathrm{T}_{\mathrm{g}}$, this reduction was more pronounced for the microparticles containing $1 \%$ PVA.
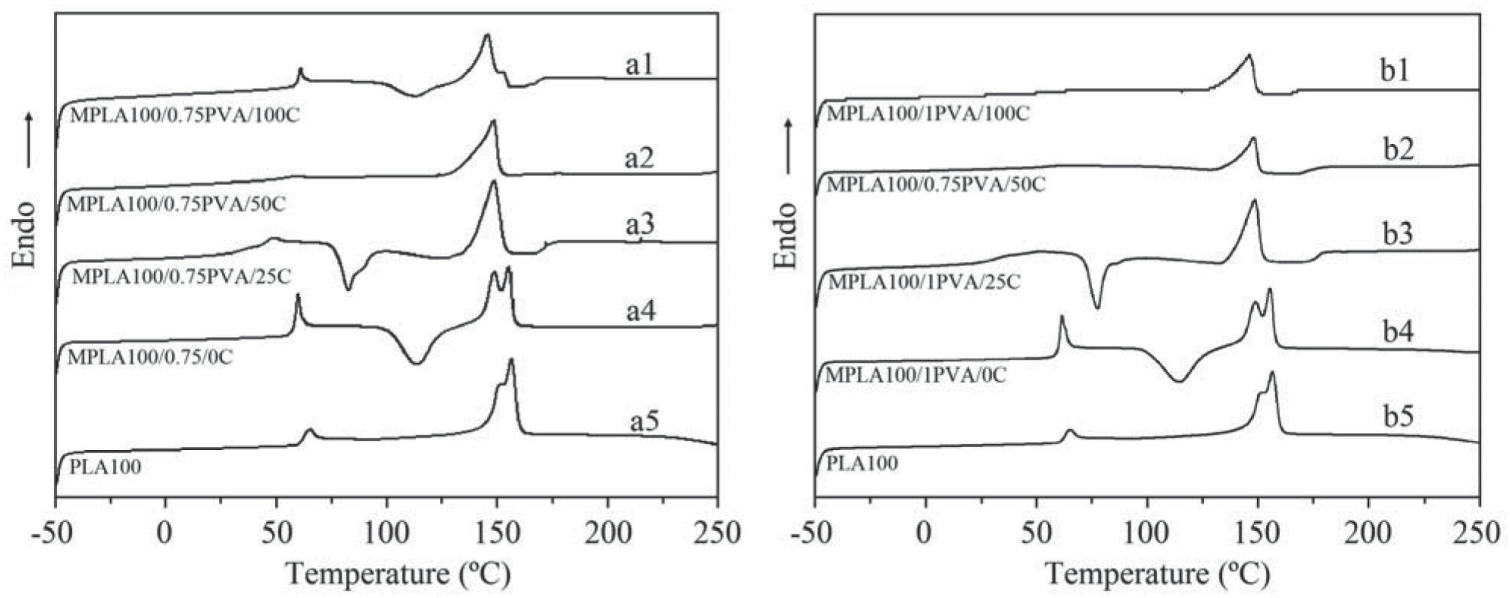

Figure 1. DSC curves at the $1^{\text {st }}$ heating for PLA100 and the microparticles (a) MPLA100/0.75PVA and (b) MPLA100/1PVA 


\section{Morphological analysis}

The morphology of the poly(lactic acid) microparticles was observed by scanning electron microscopy (SEM). The characteristics of the empty microparticles (without cardanol) were presented in a previous study. ${ }^{21}$ Completely spherical microparticles were obtained with the formulations MPLA3/1PVA/0C, MPLA100/0.75PVA/0C and MPLA100/1PVA/0C.

The micrographs of the microparticles containing 25,50 and $100 \mathrm{mg}$ of cardanol in the initial formulation, prepared with the polymer having low molar mass (PLA3), are shown in Figures 2 and 3 , respectively, for PVA concentrations of 0.75 and $1 \mathrm{wt} / \mathrm{v} \%$.

The micrographs obtained by SEM show images that seems microcapsules mainly during the process of encapsulating the cardanol for samples MPLA3/0.75PVA/25C (Figure 2b) and MPLA3/0.75PVA/50C (Figure 2c), since the polymer coating of some of the microcapsules is broken. Both samples have spherical geometry and smooth surfaces.

The breakage of the polymer coating observed in the microcapsules is probably due to the low molar mass of the used polymer, which caused the formation of a thinner coating, which might have been broken during the solvent evaporation step of the microencapsulation process. ${ }^{35}$

Sawalha, Schroën and Boom have suggested that the formation of microcapsules employing the emulsion/solvent evaporation technique is not recommended, since the microcapsules formed are too weak to withstand the direct evaporation of the solvent, possibly due to the mechanical force exerted by the gas bubbles inside the aqueous suspension. ${ }^{35}$

It is interesting to note that although the sample without cardanol (Figure 2a: MPLA3/0.75PVA/0C) did not form spherical microcapsules, ${ }^{21}$ the addition of 25 and $50 \mathrm{mg}$ of cardanol in the initial formulation led to the formation of microspheres (Figures $2 \mathrm{~b}$ and $2 \mathrm{c})$. However, for the highest cardanol concentration (100 $\mathrm{mg}$ ), no microparticles were formed (Figure 2d). This suggests that a maximum concentration limit exists able to form microparticles. Both the PVA and cardanol were able to stabilize the emulsion, and in terms of the stability of the spherical droplets dispersed during the emulsification/solvent evaporation method, the presence of cardanol had a synergistic stabilization effect. In other words, the additive facilitated formation of the microparticles. In this comparison, the similar morphology of the particles formed in the systems with $1 \mathrm{wt} / \mathrm{v} \%$ PVA without cardanol (MPLA3/1PVA/0C) ${ }^{21}$ and with $0.75 \mathrm{wt} / \mathrm{v} \%$ PVA with $50 \mathrm{mg}$ of cardanol (MPLA3/0.75PVA/50C Figure 2c) stands out. In both cases, spherical microparticles were formed. The surface morphology of the microcapsules containing cardanol and produced from the MPLA3/1PVA formulations can be observed in the SEM micrographs in Figure 3. Of the three formulations containing MPLA3/1PVA, spherical microcapsules were formed in only one (MPLA3/1PVA/25C, Figure 3b). For the samples MPLA3/1PVA/50C (Figure 3c) and MPLA3/1PVA/100C (Figure 3d), the addition of 50 and $100 \mathrm{mg}$, respectively, did not lead to the formation of microcapsules, from observing agglomerates.

The influence of the PVA concentration on the production of microcapsules was confirmed by the similar appearances of the MPLA3/0.75PVA and MPLA3/1PVA formulations as the cardanol concentration increased. In other words, there seems to be a cardanol concentration limit in the presence of PVA able to favor the formation of microcapsules. This behavior, as already mentioned, might be associated with the surfactant action of cardanol, which along with PVA stabilizes the emulsion system. The cardanol behavior as surfactant is due its amphiphilic chemical structure.

Amphiphilic substances (such as surfactants) have two regions with different polarities in their chemical structure: one polar (hydrophilic) and the other nonpolar (hydrophobic). In the case of cardanol, the phenolic group is the polar part and the hydrocarbon chain is the apolar part. This explains its ability to act at the liquidliquid interface, reducing the interfacial tension and promoting the formation of a more stable dispersed system.

An excess of emulsifying substances in the medium can directly influence the properties of the microparticles. If the concentration is very low, the polymer droplets can agglomerate, so that few or no microparticles will be formed. On the other hand, too much emulsifier impairs the emulsion's stability due to the interaction between the additive and emulsifier. This interaction can cause an increase in the solubility of the cardanol/PLA system in the aqueous medium, so that fewer cardanol molecules remain in the internal phase (organic phase) to interact with the PLA molecules when the organic phase and aqueous phase are mixed. ${ }^{36}$

The micrographs of the microparticles containing 25, 50 and $100 \mathrm{mg}$ cardanol in the initial formulation, prepared with the poly(lactic acid) having high molar mass (PLA100), are shown in

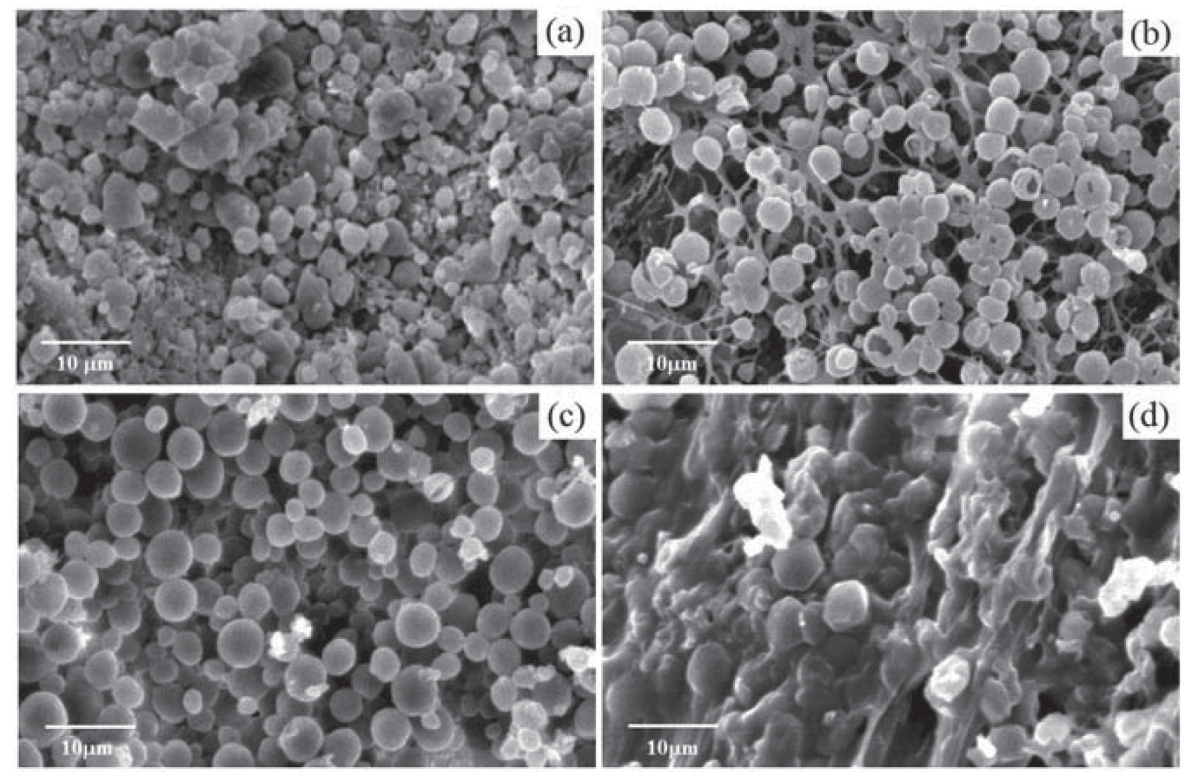

Figure 2. Micrographs of the microparticles of PLA3 containing $0.75 \mathrm{wt} / \mathrm{v} \%$ of PVA and different mass of cardanol: (a) $0 \mathrm{mg} \mathrm{g}^{21}$ (b) $25 \mathrm{mg}$; (c) $50 \mathrm{mg}$ e (d) $100 \mathrm{mg}$ 

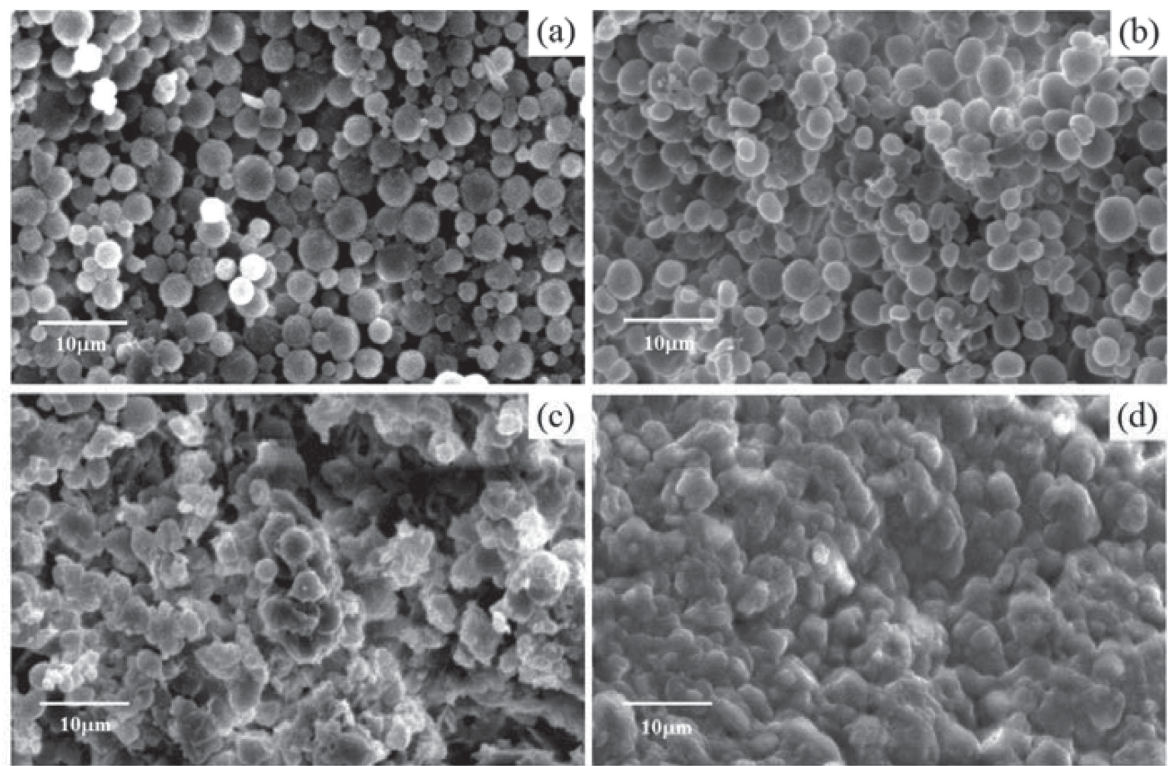

Figure 3. Micrographs of the microparticles of PLA3 containing $1 \mathrm{wt} / \mathrm{v} \%$ de PVA and different mass of cardanol: (a) $0 \mathrm{mg}^{21}$ (b) $25 \mathrm{mg}$; (c) $50 \mathrm{mg}$ e (d) $100 \mathrm{mg}$

Figures 4 and 5, respectively, for PVA concentrations of 0.75 and $1 \mathrm{wt} / \mathrm{v} \%$.

It can be seen that the microparticles produced with PLA have a smooth surface and spherical shape, and just like the microparticles produced with the polymers having low molar mass (PLA3), formed microcapsules with the use of the two PVA concentrations $(0.75$ and $1 \mathrm{wt} / \mathrm{v} \%)$. Besides this, increasing the additive's concentration in the formulation with PLA100 did not promote significant coalescence and/or agglutination between the particles, as can be seen in the microparticles of MPLA3/0.75PVA/100C, MPLA3/1PVA/50C and MPLA3/1PVA/100C (Figures 2d, 3c and 3d, respectively). This is an advantage of the PLA with high molar mass in relation to that with low molar mass. In this case, it appears that the synergistic stabilizing effect of PVA and cardanol only affected the size of the particles formed. This will be discussed further in the next section.

\section{Determination of particle size and size distribution}

The size and size distribution of the microparticles were determined after the removal of the solvent, when the particles were still in suspension. Table 4 shows the average particle diameter ( $\mathrm{v}$, $0.5)$, average volumetric diameter $(\mathrm{D}(4,3))$ and polydispersity index of the distribution curve of the microcapsules, ascertained by light scattering. The results presented in Table 4 correspond to the sizes measured by the apparatus. Table 4 does not present the results related to the MPLA3/0.75PVA/100C, MPLA3/1PVA/50C and MPLA3/1PVA/100C systems because agglomeration occurred in these samples, so no microparticles were formed, as evidenced by their respective micrographs, shown in Figures $2 \mathrm{~d}, 3 \mathrm{c}$ and $3 \mathrm{~d}$.

In general, the addition of $25 \mathrm{mg}$ of cardanol in the initial formulation led to an increase in the average diameter of the microparticles obtained, with this increase being more pronounced when using the highest PVA concentration or polymer with highest molar mass. The combined use of the high-molar-mass PLA and the highest PVA concentration enhanced the increase of the particle size. In both the system with PLA3 and $1 \mathrm{wt} / \mathrm{v} \%$ PVA and that with PLA100 and $0.75 \mathrm{wt} / \mathrm{v} \%$ PVA, the addition of $25 \mathrm{mg}$ of cardanol increased the particle size by $\sim 12 \%$ in relation to the system without
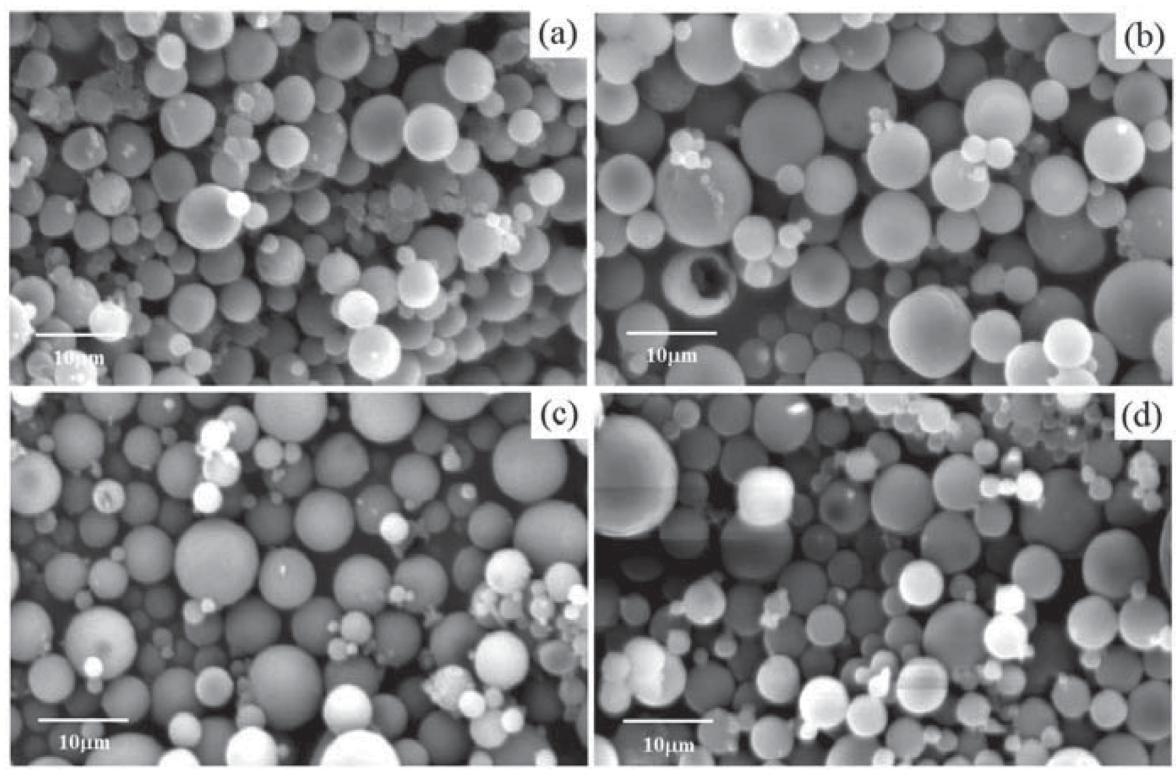

Figure 4. Micrographs of the microparticles of PLA100 containing $0.75 \mathrm{wt} / \mathrm{\nu} \%$ of PVA and different mass of cardanol: (a) $0 \mathrm{mg}^{21}$ (b) $25 \mathrm{mg}$; (c) $50 \mathrm{mg}$ e (d) $100 \mathrm{mg}$ 

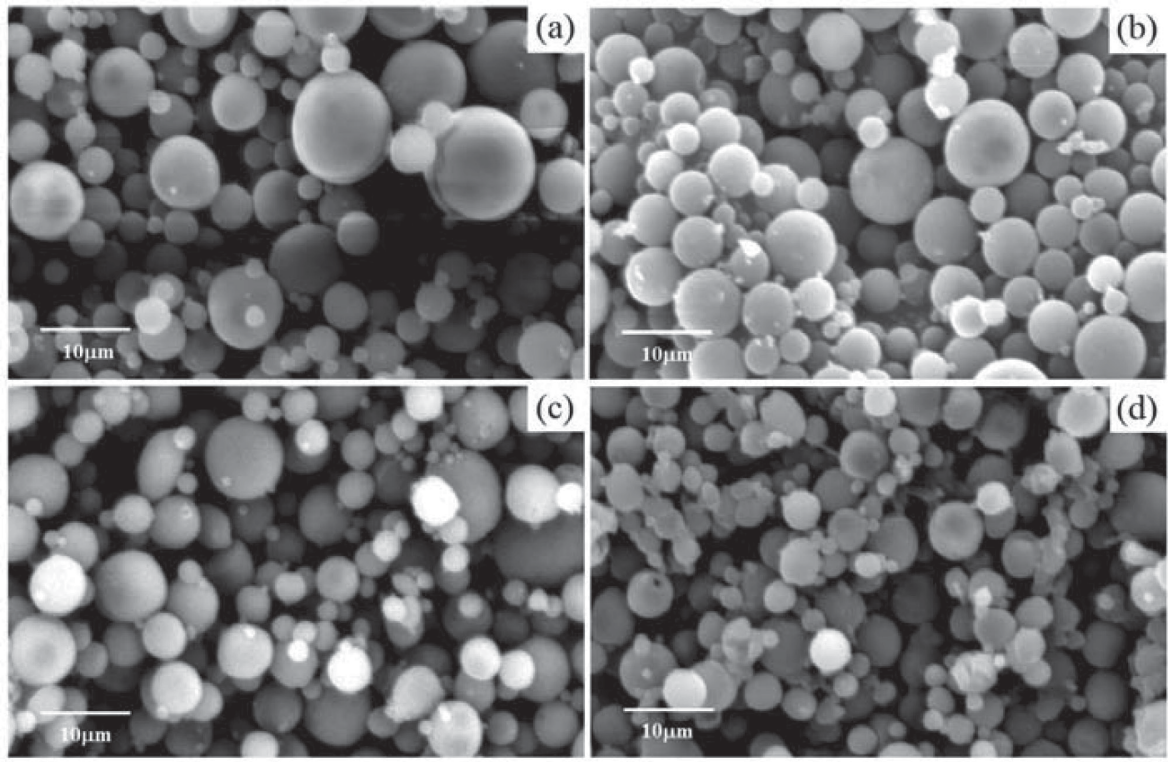

Figure 5. Micrographs of the microparticles of PLA100 containing $1 \mathrm{wt} / \mathrm{v} \%$ de PVA and different mass of cardanol : (a) $0 \mathrm{mg} \mathrm{g}^{2 \mathrm{l}}$ (b) $25 \mathrm{mg}$; (c) $50 \mathrm{mg}$ e (d) $100 \mathrm{mg}$

Table 4. Values of average particle diameter $(D(v, 0.5))$, average volumetric diameter $(D(4,3))$ and polydispersity index (span) of the microparticles

\begin{tabular}{lccc}
\hline Samples & $\begin{array}{c}\text { Average particle diameter } \\
\mathrm{D}(\mathrm{v}, 0.5)(\mu \mathrm{m})\end{array}$ & $\begin{array}{c}\text { Average volumetric diameter } \\
\mathrm{D}(4,3)(\mu \mathrm{m})\end{array}$ & $\begin{array}{c}\text { Polydispersity index } \\
\text { (Span) }\end{array}$ \\
\hline MPLA3/0.75PVA/0C34 ${ }^{21}$ & 3.04 & 5.07 & 1.51 \\
MPLA3/0.75PVA/25C & 2.80 & 2.89 & 1.01 \\
MPLA3/0.75PVA/50C & 4.11 & 9.60 & 2.73 \\
MPLA3/1PVA/0C & 21 & 3.37 & 1.43 \\
MPLA3/1PVA/25C & 2.99 & 3.59 & 1.16 \\
MPLA100/0.75PVA/0C ${ }^{21}$ & 3.33 & 4.47 & 1.95 \\
MPLA100/0.75PVA/25C & 4.14 & 5.14 & 1.89 \\
MPLA100/0.75PVA/50C & 4.66 & 5.38 & 1.90 \\
MPLA100/0.75PVA/100C & 4.94 & 7.22 & 1.67 \\
MPLA100/1PVA/0C 21 & 4.84 & 4.81 & 1.97 \\
MPLA100/1PVA/25C & 4.21 & 14.21 & \\
MPLA100/1PVA/50C & 8.48 & 12.12 & \\
MPLA100/1PVA/100C & 5.97 & 4.45 & 1.61 \\
\hline
\end{tabular}

cardanol. In turn, in the system with PLA100 and $1 \mathrm{wt} / \mathrm{v} \%$ PVA, the increase in the particle size was approximately $100 \%$ in relation to the cardanol-free system. This effect can be related to the increased viscosity of the medium, since the greater the polymer's molar mass, the greater the viscosity is of the emulsion's internal phase, making it harder to break up the droplets, thus allowing the formation of larger particles. ${ }^{37}$

The effect observed of increasing the cardanol concentration depended on the PVA concentration. At first the particle size increased when raising the cardanol concentration from 25 to 50 $\mathrm{mg}$, when using $0.75 \mathrm{wt} / \mathrm{v} \%$ PVA to produce the microparticles with both polymers. However, this behavior reversed for the highest PVA concentration $(1 \% \mathrm{wt} / \mathrm{v} \%)$ in the medium, resulting in a reduction of the average size of the microparticles with the same increase in cardanol concentration from 25 to $50 \mathrm{mg}$. Finally, the addition of $100 \mathrm{mg}$ of cardanol in the medium appeared to saturate it and reduced the average size of the microparticles to values very similar to those of the system without cardanol, as can be observed in the MPLA100/1PVA system.

\section{Encapsulation efficiency (EE)}

The encapsulation efficiency relates the quantity of the encapsulated additive with the total quantity of additive used to initiate the process. Therefore, the encapsulation efficiency (EE\%) enables verifying the concentration of cardanol in the microcapsules. To measure the encapsulation efficiency, the additive needs to be extracted from the microcapsules by fracturing them with a solvent. This solvent must be able to dissolve both the PLA and additive that is incorporated. Dichloromethane $\left(\mathrm{CH}_{2} \mathrm{Cl}_{2}\right)$ was chosen for this purpose, and also due its high volatility, low boiling point and high water immiscibility.

The response curve (absorbance vs. concentration) of cardanol in $\mathrm{CH}_{2} \mathrm{Cl}_{2}$ (y=0.0054x-0.0035) to determine the encapsulation efficiency showed a correlation coefficient of 0.999 , demonstrating linearity in the concentration range analyzed. Lyophilized microcapsules were used to calculate the EE. Table 5 reports the mean encapsulation efficiency of cardanol by the microcapsules made with MPLA3 and MPLA100, together with the respective standard deviations. 
Table 5. Values of encapsulation efficiency of cardanol by the PLA microcapsules

\begin{tabular}{lccc}
\hline Samples & Encapsulation efficiency $(\%)$ & $\begin{array}{c}\text { Standard deviation }(\%) \\
\text { Concentration of cardanol } \\
(\mu \mathrm{g} \text { cardanol/mg MP)* }\end{array}$ & $\begin{array}{c}\text { (\%) } \\
\text { MPLA3/0.75PVA/25C }\end{array}$ \\
MPLA3/0.75PVA/50C & 64.39 & \pm 3.4 & 76.84 \\
MPLA3/1PVA/25C & 38.42 & \pm 3.9 & 65.31 \\
MPLA100/0.75PVA/25C & 58.84 & \pm 7.7 & 108.9 \\
MPLA100/0.75PVA /50C & 98.14 & \pm 1.5 & 152.2 \\
MPLA100/0.75PVA /100C & 76.09 & \pm 5.4 & 266.2 \\
MPLA100/1PVA/25C & 79.95 & \pm 2.8 & 92.74 \\
MPLA100/1PVA/50C & 83.55 & \pm 11.4 & 121.4 \\
MPLA100/1PVA/100C & 60.70 & \pm 3.8 & 276.9 \\
\hline
\end{tabular}

* Mass of cardanol presents in $1 \mathrm{mg}$ of microparticles.

The encapsulation efficiency of cardanol in the MPLA3 microcapsules was lower than for the MPLA100 microcapsules. This lower efficiency might have been provoked during the solvent removal step, due to breakage of the microcapsules and consequent release of the cardanol to the medium. As stated previously, the microparticles obtained by the emulsion/solvent evaporation technique may be unable to withstand the mechanical force exerted by the gas bubbles formed inside the aqueous suspension during the solvent's evaporation. ${ }^{35}$ Besides this, PLA with lower molar mass is less able to retain and encapsulate the cardanol. This behavior can be observed in some of the systems containing MPLA3 (Figures 2d, 3c and $3 \mathrm{~d}$ ), in which higher cardanol concentration caused the formation of agglomerates.

The relatively low encapsulation efficiency of cardanol in MPLA3 microcapsules can also be associated with the solubility of cardanol in water, so that it tends to migrate to the surface of the microparticles during the process of emulsification and evaporation of the internal phase. This migration might also be facilitated to a certain degree because cardanol is an amphiphilic compound. With MPLA100 this migration process is more difficult due the PLA100 has higher viscosity.

In the MPLA3 microcapsules, the increase in the cardanol concentration promoted a reduction of the encapsulation efficiency from $64.39 \%$ in MPLA3/0.75PVA/25C to $38.42 \%$ in MPLA3/0.75PVA/50C. Besides this, the concentration of cardanol per mg of microparticles in the MPLA3/0.75PVA/25C and MPLA3/0.75PVA/50C systems (Table 5) were similar, due to saturation of the encapsulation capacity of the microparticles of MPLA/0.75PVA. However, increasing the PVA concentration from 0.75 to $1 \mathrm{wt} / \mathrm{v} \%$ did not cause a significant variation in the encapsulation efficiency or concentration. This can be observed by comparing the microparticles made with MPLA3/0.75PVA/25C and MPLA3/1PVA/25C, both of which presented encapsulation efficiency of about $60 \%$ and had similar additive concentrations, considering the standard deviations of the measures.

This migration appears not to have happened with as much facility when using polymers having higher molar mass. The increase in molar mass of the polymer promoted an increase in the encapsulation efficiency of the microparticles. For example, the EE of the MPLA3/0.75PVA/25C was $64 \%$ while it was $98 \%$ for the MPLA100/0.75PVA/25C microparticles, and the cardanol concentration in the microparticles also increased significantly (Table 5). This can probably be explained by the fact that polymers with higher molar mass (here PLA100) are able to form microparticles with stronger walls, which are less likely to break and allow cardanol to escape to the external phase than is the case for polymers with lower molar mass (e.g., PLA3). ${ }^{38}$

For the microparticles produced with PLA100, in general an increase in the PVA concentration diminished the encapsulation efficiency. However, for a high level of cardanol in the initial formulation $(100 \mathrm{mg}$ ), the efficiency results remained almost constant, varying around $80 \%$. An interesting result occurred regarding the cardanol concentrations in the PLA100 microparticles, namely that the quantity of additive to be encapsulated caused an increase in the cardanol content in the microparticles irrespective of the quantity of PVA utilized.

\section{Evaluation of the cardanol release profile of the microparticles}

Systems for controlled release of active agents are tailored to fit the release profile of a determined system. For better visualization, the release profile graphs were constructed based on the concentration of cardanol released in function of time (minutes) of exposure to the organic medium. However, some release time comparisons are carried out based on the percentage of cardanol released. To assess the influence of molar mass, PVA concentration and cardanol concentration on the release profile of the micro-encapsulated systems, we selected samples MPLA3/0.75PVA/25C, MPLA3/0.75PVA/50C, MPLA3/1PVA/25C, MPLA 100/0.75PVA/25C, MPLA $100 / 0.75 \mathrm{PVA} / 50 \mathrm{C}$, MPLA 100/0.7PVA/100C, MPLA 100/1PVA/25C and MPLA100/1PVA/50C. The tests were performed at room temperature $\left(\sim 25^{\circ} \mathrm{C}\right)$ and monitored for times of $15,30,45,60,90,120$ and 150 minutes. Despite the low absorption of PLA at the wavelength of $270 \mathrm{~nm}$, during these analyses we decided to analyze a control sample with empty microparticles in the same condition $(10 \mathrm{mg}$ in $20 \mathrm{~mL}$ of $\mathrm{CHCl}_{3}$ ). The cardanol concentration was calculated by substituting the absorbance levels obtained by UV-Vis spectrophotometry with the linear equation $(y=0.0059 x-0.0067)$, referring to the response curve of cardanol in chloroform. The percentages were then calculated from the results obtained in the test of encapsulation efficiency.

Figure 6 presents the profiles of release of the microparticles containing cardanol produced from PLA3 (low molar mass). The tests were performed in duplicate, considering the average concentration and respective standard deviation for calculation purposes. Figure 6 shows rapid release of cardanol up to 15 minutes, with concentrations of $28 \mu \mathrm{g} \mathrm{mL}^{-1}(\sim 79 \%), 36 \mu \mathrm{g} \mathrm{mL}-1$ ( 95\%) and $20 \mu \mathrm{g} \mathrm{mL}^{-1}(\sim 64 \%)$, respectively, for samples MPLA3/0.75PVA/25C, MPLA3/0.75PVA/50C and MPLA3/1PVA/25C. After 15 minutes, the amount of cardanol released remained constant with time for the samples containing $0.75 \mathrm{wt} / \mathrm{v} \%$ PVA. However, for sample 
MPLA3/1PVA/25C, containing $1 \mathrm{wt} / \mathrm{v} \%$ PVA, the controlled release continued up to 60 minutes, attributed to the formation of a more resistant wall. The cardanol concentration continued to increase with exposure time in the interval from 15 to 60 minutes, from 20.75 to $25.89 \mu \mathrm{g} \mathrm{mL}^{-1}$. After 60 minutes, no increase in cardanol concentration occurred, indicating that the maximum release had been reached.

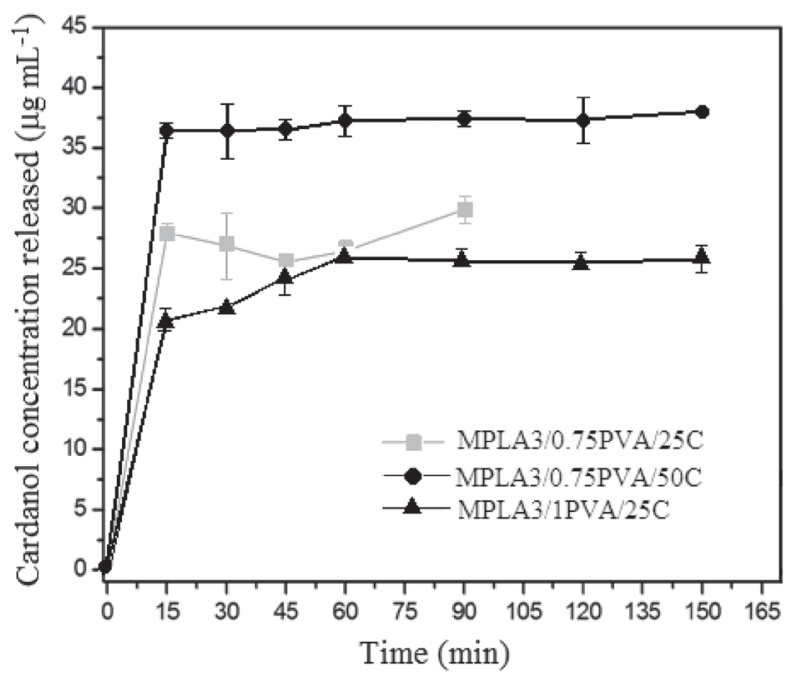

Figure 6. Cardanol release profile of the formulations MPLA3/0.75PVA/25C( $\mathbf{\square})$, MPLA3/0.75PVA/50C $(\bullet)$ and MPLA3/1PVA/25C ( $\mathbf{\Delta})$ in organic medium

The relatively faster release in the interval from 0 to 15 minutes is called the burst effect. This can be explained because cardanol is encapsulated in microcapsules whose walls also contain cardanol. The solvent first weakened the walls, causing them to rupture and release the encapsulated cardanol. In the period from 15 to 60 minutes, the release was slower, indicating that the remaining cardanol released was that contained in the walls themselves, as they dissolved. After 60 minutes, no significant increase in cardanol concentration was observed. The burst effect, normally found in microencapsulated systems, is defined as occurring when more than $50 \%$ of the active substance is released in the initial period of analysis, followed by a slower and controlled release rate thereafter, from the walls of the microparticles. ${ }^{38,39}$

Figure 7 presents the release profile graph of the microparticles in function of time for the samples containing PLA100 (high molar mass) and $0.75 \mathrm{wt} / \mathrm{v} \%$ PVA. It can be seen that in the first 15 minutes, the amounts of cardanol released were $47 \mu \mathrm{g} \mathrm{mL}^{-1}$ ( 86\%), $60 \mu \mathrm{g} \mathrm{mL}^{-1}(\sim 78 \%)$ and $119 \mu \mathrm{g} \mathrm{mL}-1$ ( 90\%) for samples MPLA100/0.75PVA/25C, MPLA100/0.75PVA/50C and MPLA100/0.75PVA/100C, respectively. These results are coherent, since the sample of microparticles with the largest quantity of cardanol (MPLA100/0.75PVA/100C) was the one that provided the greatest concentration in the release test. In the period from 15 to 60 minutes, the release was slower, from the cardanol contained in the walls of the microparticles of samples MPLA100/0.75PVA/25C and MPLA100/0.75PVA/50C. Over the period from 0 to 60 minutes, sample MPLA100/0.75PVA/50C had a slower release profile than sample MPLA100/0.75PVA/25C.

For sample MPLA100/0.75PVA/100C, the DSC/TGA analyses revealed that a high cardanol concentration makes the microparticle structure more fragile, which could explain the fast release of $90 \%$ in 15 minutes. After 60 minutes, no significant increase in the cardanol released was observed for any of the systems.

Figure 8 presents the release profile graph of the microparticles formed of PLA100 and $1 \mathrm{wt} / \mathrm{v} \%$ PVA in function of time. After

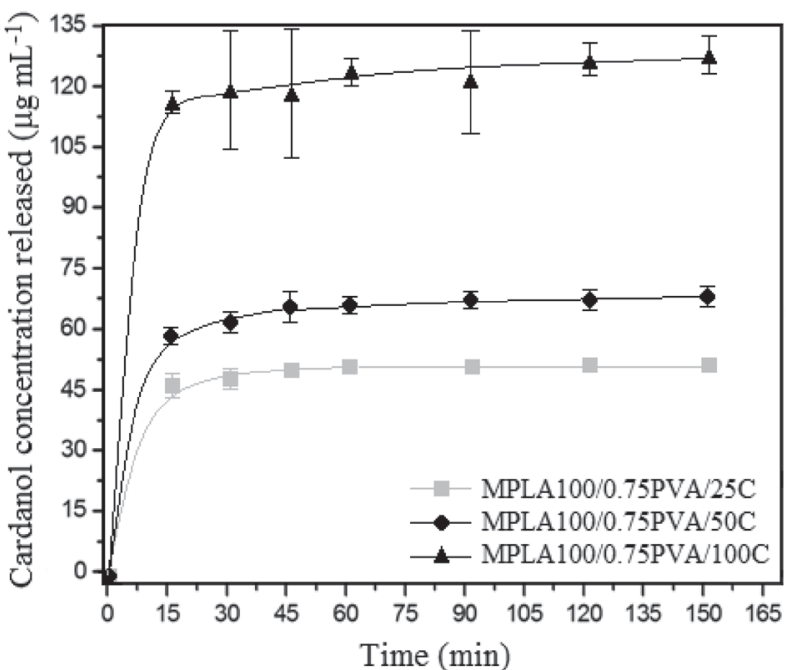

Figure 7. Cardanol release profile of the formulations MPLA100/0.75PVA/25C( $\mathbf{\square})$, MPLA100/0.75PVA/50C (-) and MPLA100/0.75PVA/100C (-) in organic medium

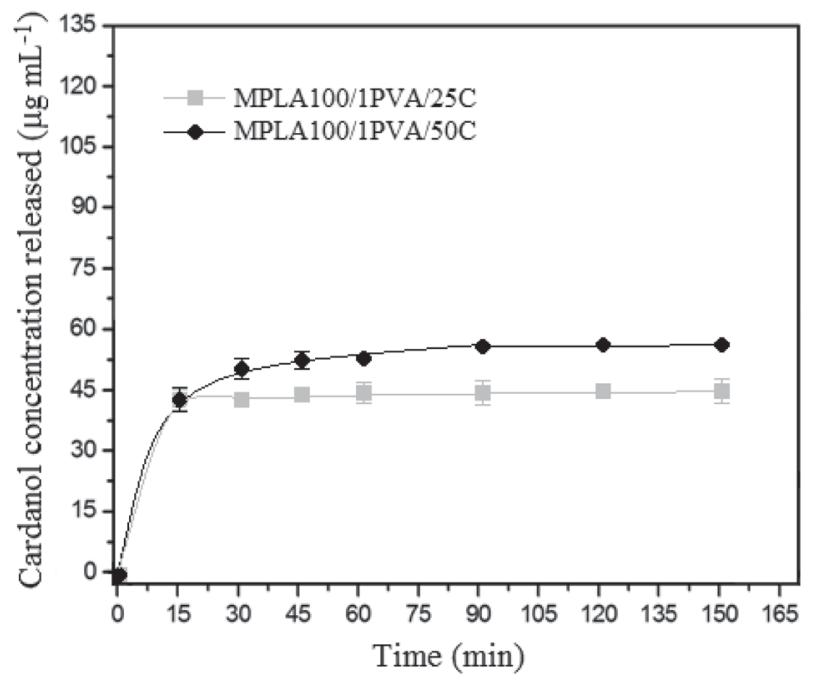

Figure 8. Cardanol release profile of the formulations MPLA100/1PVA/25C( and MPLA100/1PVA/50C (-) in organic medium

15 minutes, a burst effect was also detected, with release of $\sim 42 \mu \mathrm{g} \mathrm{mL}^{-1}$. Regarding the percentage released by the systems, about $92 \%\left(42.5 \mu \mathrm{m} \mathrm{mL}^{-1}\right)$ of the cardanol was released in the first 30 minutes from sample MPLA100/1PVA/25C, while this percentage was only attained after 90 minutes for sample MPLA100/1PVA/50C. These results are similar to those obtained using $0.75 \mathrm{wt} / \mathrm{v} \%$ PVA (Figure 7), in which the sample containing $50 \mathrm{mg}$ of cardanol showed slower release.

The influence of molar mass on the release profile of the microparticles can be observed in Figure 9. The microparticles prepared with the polymer having higher molar mass (MPLA100/1PVA/25C) showed slower and longer-lasting release of cardanol than those produced with the polymer having lower molar mass (MPLA3/1PVA/25C). This can be attributed to the fact that high molar mass can make the microparticles' walls more resistant, as also observed in the TGA results. Besides this, the fact that the microparticles produced with PLA100 were larger on average than those produced with PLA3 might have contributed to the slower release, since the larger the diameter of the particles, the smaller the surface area exposed to the medium is. ${ }^{40}$ 


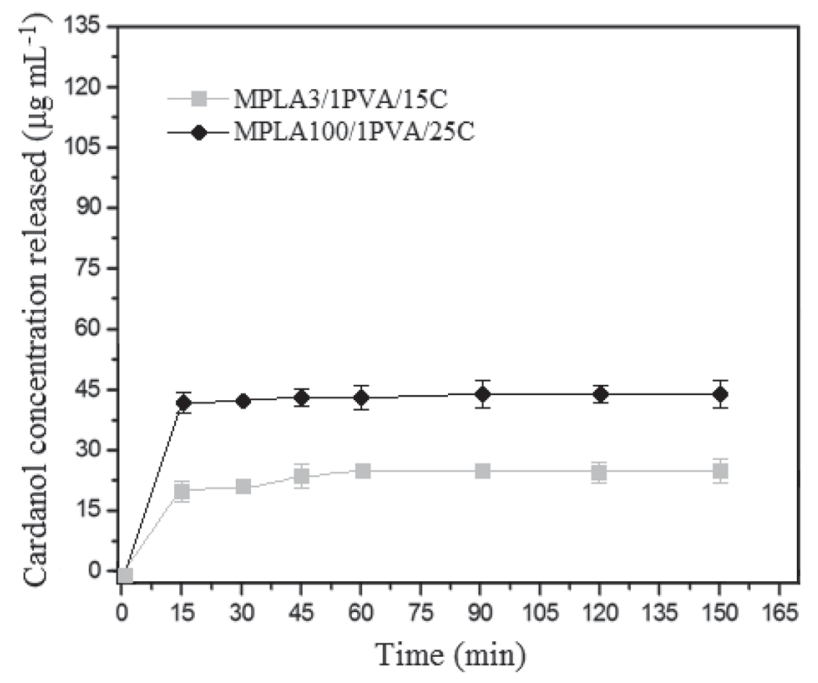

Figure 9. Cardanol release profile with $1 \mathrm{wt} / \mathrm{\%}$ of PVA from PLA3 (ם) and PLA100 (O) microparticles

The influence of the PVA concentration on the release profile can be seen in Figure 10. This influence is related to the quantity of cardanol in the formulation. Two distinct behavior patterns were observed regarding the effect of PVA concentration on the microparticles' release profile. When the amount of cardanol initially incorporated was low (25 mg) (Figure 10a), the increase in PVA concentration from 0.75 to $1 \mathrm{wt} / \mathrm{v} \%$ did not cause a significant change in the release profile. For the systems produced initially with $50 \mathrm{mg}$ of cardanol (Figure 10b), the release speed declined with increase of the PVA concentration. Increasing the PVA from 0.75 to $1 \mathrm{wt} / \mathrm{v} \%$ caused a reduction in the percentage of the additive released from the microparticles (Figure 10b). Again, a burst effect was observed in the first 15 minutes, followed by slower release of cardanol from 15 to 90 minutes for both systems tested. After 90 minutes, the concentration of the additive remained constant.

\section{CONCLUSION}

The findings indicate that the efficiency in encapsulation the chemical additive depends on the molar mass of the PLA, the type and concentration of the emulsifier (PVA) and the type and concentration of the additive to be encapsulated. The addition of cardanol during the production of the microparticles promoted an increase in their diameter, both for those obtained from the polymers with low and high molar mass (MPLA3 and MPLA100), with this increase being dependent on the cardanol concentration. The existence of interaction between the cardanol and polymer matrix was proved, since we observed a shift of the endothermic event related to the melting of the PLA. The cardanol also acted as a stabilizer in the process of producing the microparticles. The formulations showed a pronounced burst effect in the first $15 \mathrm{~min}$ of release. Afterward, some formulations presented a slower and more sustained release of cardanol up to 90 minutes, with the amount released remaining constant until the end of the test at 150 minutes. The MPLA100/1PVA/50C microparticles had the slowest release profile among the formulations tested.

\section{ACKNOWLEDGMENTS}

The authors thank the Brazilian agencies for the financial support: CNPq (307193/2016-0), CAPES and FAPEJ (E-26/201.233/2014).
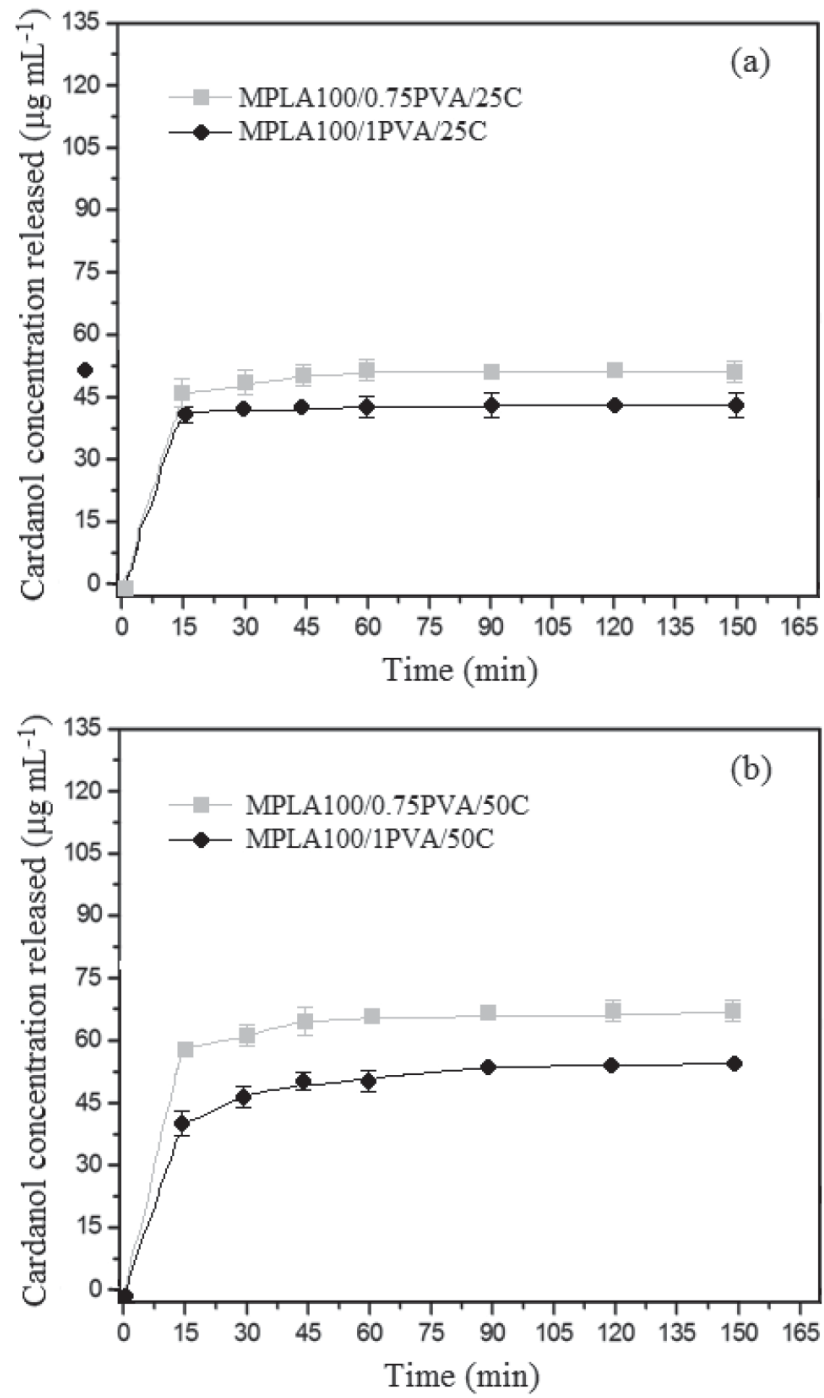

Figure 10. Cardanol release profile from microparticles PLA100 with 0.75 $w t / v \%$ and $1.0 \mathrm{wt} / \mathrm{v} \%$ of PVA: (a) $25 \mathrm{mg}$ and (b) $50 \mathrm{mg}$ of cardanol

\section{REFERENCES}

1. Risfaheri, I.; Irawadi, T. T.; Nur, M. A.; Sailah, I.; Indones. J. Agric. 2009, 2, 11.

2. Darromana, E.; Durandb, N.; Boutevina, B.; Caillola, S.; Prog. Org. Coatings 2016, 91, 9.

3. Mazzetto, S. E.; Lomonaco, D.; Mele, G.; Quim. Nova 2009, 32, 732.

4. Moreira, L. F. B.; González, G.; Lucas, E. F.; Polímeros 1998, 8, 46.

5. Rocha Junior, L.C.; Ferreira, M. S.; Ramos, A. C. S.; J. Pet. Sci. Eng. 2006, 51, 26.

6. Moreira, L. F. B.; González, G.; Lucas, E. F.; J. Appl. Polym. Sci. 1999, $73,29$.

7. Lima, A. F.; Mansur, C. R. E.; Lucas, E. F.; González, G.; Energy Fuels 2010, 24, 2369.

8. Ferreira, S. R.; Louzada, H. F.; Dip, R. M. M.; Gonzalez, G.; Lucas, E. F.; Energy Fuels 2015, 29, 7213

9. Ramalho, J. B. V. S.; Lechuga, F. C.; Lucas, E. F.; Quim. Nova 2010, 33, 1664.

10. Honse, S. O.; Ferreira, S. R.; Mansur, C. R. E.; Gonzalez, G.; Lucas, E. F.; Quim. Nova 2012, 35, 1991.

11. Lucas, E. F.; Ferreira, L. S.; Khalil, C. N. Em Encyclopedia of Polymer Science and Technolology; Mark, H. F., ed.; John Wiley \& Sons: New York, 2015 . 
12. Lucas, E. F.; Mansur, C. R. E.; Spinelli, L.; Queirós, Y. G. C.; Pure Appl. Chem. 2009, 81, 476.

13. Spinelli, L. S.; Louvisse, A. M. T.; Lucas, E. F.; Pet. Sci. Technol. 2006, 24,61 .

14. Spinelli, L. S.; Aquino, A. S.; Pires, R. V.; Barbosza, E. M.; Louvisse, A. M. T.; Lucas, E. F.; J. Pet. Sci. Eng. 2007, 58, 111.

15. Powell, R.J.; Fischer, A. R.; Gdanski, R. D.; McCabe, M. A.; Pelly, S. D.; Soc. Pet. Eng. (1995), doi:10.2118/30700-MS.

16. Reddy, B. R.; Crook, R. J.; Chatterji, J.; King, B. J.; Gray, D. W.; Fitzgerald, R. M.; Powell, R. J.; Todd, B. L.; US pat. 6,209,646 2001.

17. Cao-Hoanga, L.; Fougèrea, R.; Wachéa, Y.; Food Chem. 2011, 124, 42.

18. Koppolua, B.P.; Smith, S. G.; Ravindranathan, S.; Jayanthi, S.; Suresh Kumar, T. K.; Zaharoff, D. A.; Biomaterials 2014, 35, 4382.

19. Gama, R. M.; Balogh, T. S.; França, S.; Dias, T. C. S.; Bedin, V.; Baby, A. R.; Matos, J. R.; Velasco, M. V. R.; Therm. Anal. Calorim. 2011, 106, 399.

20. Li, J.; Wang, H.; Baixue, Y.; Xu, L.; Zheng, N.; Chen, H.; Sanming, L.; Mater. Sci. Eng. C 2016, 58, 273.

21. Cardoso, J. J. F.; Ricci-Júnior, E.; Queirós, Y. G.; Gentili, D.; Lucas, E. F.; J. Therm. Anal. Calorim. 2016, 126, 1349.

22. Srivastava, A.; Vivek, M.; Singh, P.; Srivastava, A.; Kumar, R.; J. Therm. Anal. Calorim. 2012, 107, 211.

23. Martins, J.; Wilson, R.; Rosencrance, S.; Previs, D.; US pat 8,236,889 B2 2012

24. Reddy, B. R.; Boul, P. J.; Agashe, S. S.; US pat. 8,540,025 2014.

25. Mirakyan, A.; Hutchins, R. D.; Williamson, D.; Clum, E.; US WO2012/125890 A2 2012.

26. Parker, M. A.; Laramay, S. B.; Soc. Pet. Eng. (1992), doi: 10.2118/24300-MS
27. Chen, C. C.; Chueh, J. Y.; Tseng, H.; Huang H. M.; Lee, S. Y.; Biomaterials 2003, 24, 1167.

28. Jamshidian, M.; Tehrany, E. A.; Imran, M.; Compr. Rev. Food Sci. Food Saf. 2010, 9, 552.

29. Felfel, R. M.; Hossain, K. M. Z.; Parsons, A. J.; Rudd, C. D.; Ahmed, I.; J. Mater. Sci. 2015, 50, 3942.

30. Zeng, R.-C.; Qui, W.-C.; Song, Y.-W.; He, Q.-K.; Chui, H.-Z.; Han, E.H.; Front. Mater. Sci. 2014, 8, 343.

31. Suave, J.; Dallagnol, E. C.; Pezzin, A. P. T.; Silva, D. A. K.; Meier, M. M.; Soldi, V.; Revista Saúde e Ambiente 2006, 7, 12.

32. Cardoso, J. J. F.; Queirós, Y. G. C.; Machado, K. J. A.; Costa, J. M.; Lucas, E. D. Brazilian Journal of Petroleum and Gas 2013, 7, 57.

33. Malvern Mastersizer 2000 user manual, http://www.malvern.com/en/ support/resource-center/user-manuals/MAN0384EN.aspx, acessado em novembro de 2017.

34. Türk, C. T.; Hasçiçek, C.; Gönül, N.; J. Therm. Anal. Calorim. 2009, 95, 865.

35. Sawalha, H.; Schroën, K.; Boom, K.; Chem. Eng. J. 2011, 169, 1.

36. Hans, M. L.; Lowman, A. M.; Curr. Opin. Solid State Mater. Sci. 2002, $6,319$.

37. Fu, X.; Ping, Q.; Gao, Y.; J. Microencapsulation 2005, 22, 705.

38. Yeo, Y.; Park, K.; Arch. Pharm. Res. 2004, 27, 1.

39. Esser-Kahn, A. P.; Odom, S. A.; Sottos, S. R. W.; Moore, J. S.; Macromolecules 2011, 44, 5539.

40. Ghassemi, A. H.; Steenbergen, M. J. V.; Talsma, H.; Nostrum, C. F. V.; Crommelin, D. J. D.; Hennink, W. E.; Pharm. Res. 2010, 27, 2008. 